\begin{tabular}{|c|l|}
\hline Title & $\begin{array}{l}\text { Josephson current through superconductor/diffusive normal-metal superconductor junctions: Interference effects } \\
\text { governed by pairing symmetry }\end{array}$ \\
\hline Author(s) & A sano, Y asuhiro; Tanaka, Y ukio; Y okoy ama, Takehito; Kashiway a, Satoshi \\
\hline Citation & $\begin{array}{l}\text { Physical Review B, 74(064507) } \\
\text { https://doi.org/10.1103/PhysRevB.74.064507 }\end{array}$ \\
\hline Issue Date & 2006 \\
\hline Doc URL & http://hdl.handle.net/2115/14740 \\
\hline Rights & Copyright $\odot$ 2006 A merican Physical Society \\
\hline Type & article \\
\hline File Information & PRB2006-74-6.pdf \\
\hline
\end{tabular}

Instructions for use 


\title{
Josephson current through superconductor/diffusive-normal-metal/superconductor junctions: Interference effects governed by pairing symmetry
}

\author{
Yasuhiro Asano* \\ Department of Applied Physics, Hokkaido University, Sapporo 060-8628, Japan \\ Yukio Tanaka and Takehito Yokoyama \\ Department of Applied Physics, Nagoya University, Nagoya 464-8603, Japan \\ and CREST, Japan Science and Technology Corporation (JST) Nagoya, 464-8603, Japan \\ Satoshi Kashiwaya \\ National Institute of Advanced Industrial Science and Technology, Tsukuba 305-8568, Japan
}

(Received 17 April 2006; published 17 August 2006)

\begin{abstract}
The Josephson effect in superconductor/diffusive-normal-metal/superconductor junctions is studied numerically by using the recursive Green function method. In superconductors, we consider spin-singlet $s$ - and $d$-wave and spin-triplet $p$-wave pairing symmetries. The pairing symmetry governs two interference effects in junctions: the formation of a midgap Andreev resonant state at junction interfaces and the proximity effect in diffusive normal metals. A cooperative effect between the two interference effects causes anomalous Josephson current in a $p$-wave symmetry.
\end{abstract}

DOI: 10.1103/PhysRevB.74.064507

PACS number(s): 74.50.+r, 74.25.Fy, 74.70.Tx

\section{INTRODUCTION}

The Josephson effect is the highlight of superconducting phenomena. ${ }^{1}$ The gradient in the macroscopic phase of superconductivity drives electric currents in equilibrium. A considerable number of theoretical studies have revealed the nature of the Josephson effect ${ }^{2}$ since a Josephson current formula in superconductor/insulator/superconductor (SIS) junctions was derived based on a microscopic theory. ${ }^{3}$ The Josephson current is also observed in superconductor/ diffusive-normal-metal/superconductor (SNS) junctions, where the length of a normal metal is much larger than the mean free path due to elastic impurity scatterings. ${ }^{2,4}$ In SNS junctions, the Josephson effect is understood in terms of the proximity effect; Cooper pairs penetrate into diffusive normal metals. In this sense, supercurrents flow as far as the amplitudes of the Cooper pairs are finite in diffusive normal metals. The applicability of these theories, however, is limited to superconducting junctions of conventional $s$-wave pairing symmetry.

In recent years, transport phenomena in unconventional superconductors have attracted considerable interest because high- $T_{c}$ superconductivity is characterized by $d$-wave pairing symmetry. ${ }^{5-7}$ Contrary to $s$-wave symmetry, the pair potentials in unconventional superconductors change their sign on the Fermi surface. It is now known that the sign change of pair potentials causes the formation of a midgap Andreev resonant state (MARS) at the surface of superconductors ${ }^{8-12}$ because of the interference effect of a quasiparticle. ${ }^{13}$ The MARS spatially localizes at a distance of $\xi_{0}=\hbar v_{F} / \pi \Delta_{0}$ from the surface ${ }^{14}$ and energetically forms just on the Fermi energy, where $\xi_{0}$ is the coherence length, $v_{F}=\hbar k_{F} / m$ is the Fermi velocity, $k_{F}$ is the Fermi wave number, and $m$ is the mass of an electron. The low-temperature anomaly of the Josephson current in SIS junctions of unconventional superconductors ${ }^{15-25}$ is a consequence of the resonant tunneling of Cooper pairs through the MARS.
The sign change of pair potentials affects also the proximity effect in diffusive normal metals. Suppression of the proximity effect is usually expected because the wave function of a Cooper pair originating from the positive part of pair potentials cancels that originating from the negative part. In fact, one of authors has shown that the ensemble average of the Josephson current vanishes in diffusive SNS junctions for several unconventional superconductors. ${ }^{26,27}$ This phenomenon is now interpreted as the absence of the proximity effect in diffusive normal metals. ${ }^{28,29}$ On the other hand, two of us discussed anomalous enhancement of the zero-bias tunneling conductance due to the proximity effect in the presence of the MARS. ${ }^{30,31}$

In this paper, we study the ensemble average of the Josephson current in SNS junctions of unconventional superconductors. The Josephson current in a single sample is calculated numerically by using the recursive Green function method. ${ }^{32-34}$ After calculating the Josephson current for a number of SNS junctions with different impurity configurations, the ensemble average and the fluctuations are obtained. We consider spin-singlet $s$ - and $d$-wave and spin-triplet $p$-wave pairing symmetries in superconductors. The Josephson effect depends strongly on the pairing symmetries because the two interference effects (the formation of the MARS and the proximity effect) are sensitive to pairing symmetries. We show that a cooperative effect between the midgap Andreev resonant states and the proximity effect causes anomalous Josephson current in a $p$-wave symmetry. A part of this paper has been already published elsewhere. ${ }^{35}$

This paper is organized as follows. In Sec. II, we discuss a relation between the pairing symmetry of superconductivity and the two interference effects of quasiparticles: the proximity effect and the MARS. The theoretical model and the method of simulation are explained in Sec. III. Numerical results of the Josephson current are discussed for four pairing symmetries in Sec. IV. The origin of the anomalous Joseph- 
son effect in the $p_{x}$-wave symmetry is explained in Sec. IV. In Sec. VI, we summarize the paper. Throughout this paper, we take units of $\hbar=k_{B}=1$, where $k_{B}$ is the Boltzmann constant.

\section{PAIRING SYMMETRIES}

We consider five pairing symmetries in two-dimensional superconductors as follows:

$$
\begin{gathered}
\Delta_{k}^{(s)}=\Delta_{0}, \\
\Delta_{k}^{\left(d_{\left.x^{2}-y^{2}\right)}=\right.} \Delta_{0}\left(\bar{k}_{x}^{2}-\bar{k}_{y}^{2}\right), \\
\Delta_{k}^{\left(d_{x y}\right)}=\Delta_{0} 2 \bar{k}_{x} \bar{k}_{y}, \\
\Delta_{k}^{\left(p_{y}\right)}=\Delta_{0} \bar{k}_{y}, \\
\Delta_{k}^{\left(p_{x}\right)}=\Delta_{0} \bar{k}_{x},
\end{gathered}
$$

where $\bar{k}_{x}=k_{x} / k_{F}$ and $\bar{k}_{y}=k_{y} / k_{F}$ are the normalized wave numbers on the Fermi surface in the $x$ and $y$ directions, respectively. In this section, we assume an isotropic Fermi surface in two-dimensional electron systems. Thus the relation $\vec{k}_{x}^{2}$ $+\vec{k}_{y}^{2}=1$ holds. The electric current is parallel to the $x$ direction and the junction interface is parallel to the $y$ direction as shown in Fig. 1(a). In Eqs. (1)-(5), we only consider the orbital part of the pair potentials. The spin part will be taken into account later on. The pair potentials in momentum space are illustrated in Fig. 1(b). The pair potential $\Delta_{k}^{\left(d_{\left.x^{2}-y^{2}\right)}\right)}$ is realized in high- $T_{c}$ superconductor junctions in which the $a$ axis of high- $T_{c}$ compound is set to be parallel to the $x$ direction. When the $a$ axis is oriented by $45^{\circ}$ from the $x$ direction, the pair potential is described by $\Delta_{k}^{\left(d_{x y}\right)}$. The $p$-wave symmetry is a possible candidate in organic superconductors, ${ }^{36-38}$ $\mathrm{Sr}_{2} \mathrm{RuO}_{4},{ }^{39}$ and heavy-fermion superconductors.

The constructive interference of a quasiparticle enables the formation of the MARS at junction interfaces when a relation

$$
\Delta_{k_{x}, k_{y}} \Delta_{-k_{x}, k_{y}}<0
$$

is satisfied. ${ }^{11,13}$ The pair potentials in Eqs. (3) and (5) satisfy Eq. (6) for all wave numbers. Actually, the low-temperature anomaly of the Josephson current has been reported ${ }^{15-18,24,25}$ in SIS junctions of these symmetries.

The absence of the proximity effect in diffusive normal metals is described by a relation ${ }^{26-28}$

$$
\Delta_{k_{x}, k_{y}}=-\Delta_{k_{x},-k_{y}} .
$$

The pair potentials in Eqs. (3) and (4) satisfy Eq. (7). In Fig. $1(\mathrm{~b})$, we classify the pairing symmetries into four groups by the presence $(\circ)$ or absence $(X)$ of the two interference

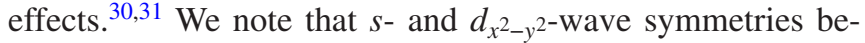
long to the same group.

\section{MODEL}

Let us consider superconductor/normal-metal/ superconductor junctions of the two-dimensional tight-

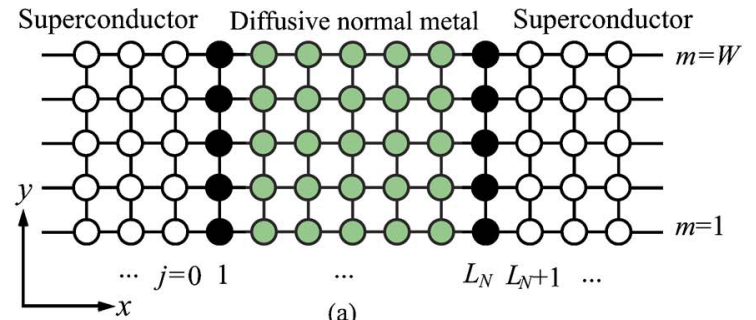

(a)

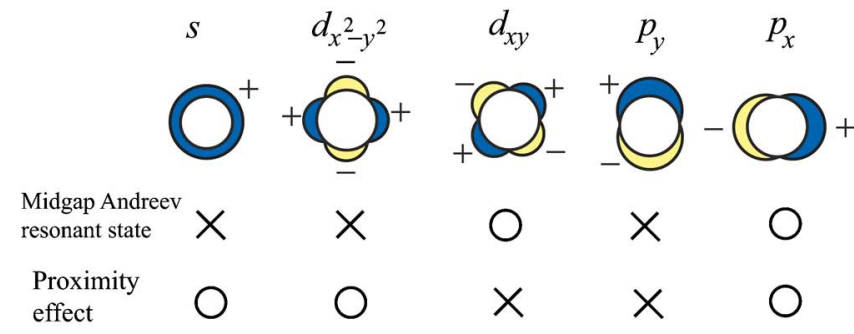

(b)

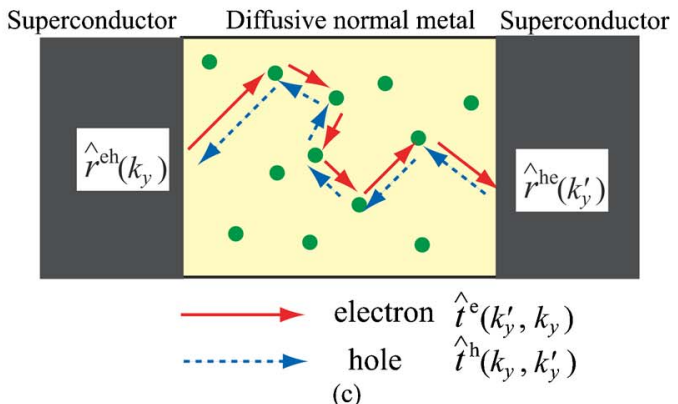

FIG. 1. (Color online) A schematic figure of SNS junctions of the tight-binding model is shown in (a). We illustrate the pair potentials in momentum space in (b). In (c), a propagation process in a diffusive normal metal is illustrated, where solid circles represent impurities.

binding model as shown in Fig. 1(a). A vector $\boldsymbol{r}=j \boldsymbol{x}+m \boldsymbol{y}$ points to a lattice site, where $\boldsymbol{x}$ and $\boldsymbol{y}$ are unit vectors in the $x$ and $y$ directions, respectively. The junction consists of three regions: a normal metal (i.e., $1 \leq j \leq L_{N}$ ) and two superconductors (i.e., $-\infty \leq j \leq 0$ and $L_{N}+1 \leq j \leq \infty$ ). In the $y$ direction, we assume the periodic boundary condition. The number of lattice sites in the $y$ direction is $W$. Electronic states in superconducting junctions are described by the mean-field Hamiltonian

$$
\begin{aligned}
& H_{\mathrm{BCS}}=\frac{1}{2} \sum_{\boldsymbol{r}, \boldsymbol{r}^{\prime}}\left[\widetilde{c}_{\boldsymbol{r}}^{\dagger} h_{\boldsymbol{r}, \boldsymbol{r}^{\prime}} \widetilde{c}_{\boldsymbol{r}^{\prime}}-\widetilde{c}_{\boldsymbol{r}}^{t} h_{\boldsymbol{r}, \boldsymbol{r}^{\prime}}^{*}\left\{\widetilde{c}_{\boldsymbol{r}^{\prime}}^{\dagger}\right\}^{t}\right]+\frac{1}{2} \sum_{\boldsymbol{r}, \boldsymbol{r}^{\prime} \in \mathrm{S}}\left[\widetilde{c}_{\boldsymbol{r}}^{\dagger} \hat{\Delta}_{\boldsymbol{r}, \boldsymbol{r}^{\prime}}\left\{\tilde{c}_{\boldsymbol{r}^{\prime}}^{\dagger}\right\}^{t}\right. \\
& \left.-\left\{\widetilde{c}_{r}\right\}^{t} \hat{\Delta}_{r, r^{\prime}}^{*} \widetilde{r}_{r^{\prime}}\right], \\
& h_{r, r^{\prime}}=-t \delta_{\left|r-r^{\prime}\right|=1}+\left(\epsilon_{r}-\mu+4 t\right) \delta_{r, r^{\prime}},
\end{aligned}
$$

$$
\hat{\Delta}_{\boldsymbol{r}, \boldsymbol{r}^{\prime}}=e^{i \varphi_{j}} \times\left\{\begin{array}{cc}
i \boldsymbol{d}_{\boldsymbol{r}, \boldsymbol{r}^{\prime}} \cdot \hat{\boldsymbol{\sigma}} \hat{\sigma}_{2}: & \text { triplet }, \\
i d_{\boldsymbol{r}, \boldsymbol{r}^{\prime}} \hat{\sigma}_{2}: & \text { singlet },
\end{array}\right.
$$




$$
\widetilde{c}_{r}=\left(\begin{array}{c}
c_{r, \uparrow} \\
c_{r, \downarrow}
\end{array}\right)
$$

where $c_{r, \sigma}^{\dagger}\left(c_{r, \sigma}\right)$ is the creation (annihilation) operator of an electron at $\boldsymbol{r}$ with spin $\sigma=(\uparrow$ or $\downarrow)$, "S" in the summation means superconductors, and $\hat{\sigma}_{j}$ with $j=1-3$ are the Pauli matrices. In superconductors, $\epsilon_{r}$ is taken to be zero. Macroscopic phases are given by $\varphi_{j}=\varphi_{L}$ in the left superconductor and by $\varphi_{j}=\varphi_{R}$ in the right one. The hopping integral $t$ is considered among the nearest-neighbor sites. We assume that $t$ and the Fermi energy $\mu$ are common in superconductors and normal metals. In normal metals, the on-site potential is given randomly in the range of $-V_{I} / 2 \leq \epsilon_{r} \leq V_{I} / 2$. We also introduce the insulating barrier at $j=1$ and $j=L_{N}$, where $\epsilon_{r}$ is given by $V_{B}$. The pair potentials corresponding to Eqs. (1)-(5) are defined in real space,

$$
\begin{gathered}
d_{\boldsymbol{r}, \boldsymbol{r}^{\prime}}^{(s)}=\Delta \delta_{j, j^{\prime}} \delta_{m, m^{\prime}}, \\
d_{\boldsymbol{r}, \boldsymbol{r}^{\prime}}^{\left(d_{\left.x^{2}-y^{2}\right)}\right)}=\frac{\Delta}{2}\left(\delta_{\left|j-j^{\prime}\right|, 1} \delta_{m, m^{\prime}}-\delta_{j, j^{\prime}} \delta_{\left|m-m^{\prime}\right|, 1}\right), \\
d_{\boldsymbol{r}, \boldsymbol{r}^{\prime}}^{\left(d_{y^{\prime}}\right)}=\frac{\Delta}{2} \operatorname{sgn}\left(j-j^{\prime}\right) \operatorname{sgn}\left(m-m^{\prime}\right) \delta_{\left|r-\boldsymbol{r}^{\prime}\right|, \sqrt{2},}, \\
\boldsymbol{d}_{\boldsymbol{r}, \boldsymbol{r}^{\prime}}^{\left(p_{y}\right)}=\frac{\Delta}{2} \operatorname{sgn}\left(m-m^{\prime}\right) \delta_{\left|m-m^{\prime}\right|, 1} \delta_{j, j^{\prime}} \boldsymbol{e}, \\
\boldsymbol{d}_{\boldsymbol{r}, \boldsymbol{r}^{\prime}}^{\left(p_{x}\right)}=\frac{\Delta}{2} \operatorname{sgn}\left(j-j^{\prime}\right) \delta_{\left|j-j^{\prime}\right|, 1} \delta_{m, m^{\prime}} \boldsymbol{e},
\end{gathered}
$$

where $\boldsymbol{e}$ is a unit vector in the spin space. The amplitude of the pair potential at a finite temperature is denoted by $\Delta$. We describe the dependence of $\Delta$ on temperatures by the BCS theory. When we calculate the Josephson current, a macroscopic phase factor $e^{i \varphi_{L}}\left(e^{i \varphi_{R}}\right)$ must be multiplied by Eqs. (12)-(16) for a superconductor on the left- (right-) hand side. In numerical simulations, we choose $\boldsymbol{e}=\boldsymbol{e}_{3}$ in the $p$-wave symmetries because the following argument is independent of the spin directions of a Cooper pair.

The Hamiltonian is diagonalized by the Bogoliubov transformation

$$
\begin{gathered}
{\left[\begin{array}{c}
\tilde{c}_{\boldsymbol{r}} \\
\left\{\tilde{c}_{\boldsymbol{r}}^{\dagger}\right\}^{t}
\end{array}\right]=\sum_{\lambda}\left[\begin{array}{ll}
\hat{u}_{\lambda}(\boldsymbol{r}) & \hat{v}_{\lambda}^{*}(\boldsymbol{r}) \\
\hat{v}_{\lambda}(\boldsymbol{r}) & \hat{u}_{\lambda}^{*}(\boldsymbol{r})
\end{array}\right]\left[\begin{array}{c}
\tilde{\gamma}_{\lambda} \\
\left\{\tilde{\gamma}_{\lambda}^{\dagger}\right\}^{t}
\end{array}\right]} \\
\tilde{\gamma}_{\lambda}=\left(\begin{array}{l}
\gamma_{\lambda, \uparrow} \\
\gamma_{\lambda, \downarrow}
\end{array}\right),
\end{gathered}
$$

where $\gamma_{\lambda, \sigma}^{\dagger}\left(\gamma_{\lambda, \sigma}\right)$ is the creation (annihilation) operator of a Bogoliubov quasiparticle. The wave functions, $\hat{u}_{\lambda}$ and $\hat{v}_{\lambda}$, satisfy the Bogoliubov-de Gennes (BdG) equation ${ }^{40}$

$$
\sum_{\boldsymbol{r}^{\prime}}\left[\begin{array}{cc}
h_{\boldsymbol{r}, \boldsymbol{r}^{\prime}} \hat{\sigma}_{0} & \hat{\Delta}_{\boldsymbol{r}, \boldsymbol{r}^{\prime}} \\
-\hat{\Delta}_{\boldsymbol{r}, \boldsymbol{r}^{\prime}}^{*} & -h_{\boldsymbol{r}, \boldsymbol{r}^{\prime}}^{*} \hat{\sigma}_{0}
\end{array}\right]\left[\begin{array}{l}
\hat{u}_{\lambda}\left(\boldsymbol{r}^{\prime}\right) \\
\hat{v}_{\lambda}\left(\boldsymbol{r}^{\prime}\right)
\end{array}\right]=E_{\lambda}\left[\begin{array}{l}
\hat{u}_{\lambda}(\boldsymbol{r}) \\
\hat{v}_{\lambda}(\boldsymbol{r})
\end{array}\right] .
$$

The eigenvalue $E_{\lambda}$ is independent of spin channels because we consider unitary states in superconductors. Here we briefly explain the method to calculate the Josephson current for the $d_{x^{2}-y^{2}}$-wave symmetry. The application to other symmetries is straightforward. The BdG equation in Eq. (19) for the $d_{x^{2}-y^{2}}$-wave symmetry is decoupled into two equations

$$
\sum_{\boldsymbol{r}^{\prime}}\left[\begin{array}{cc}
h_{\boldsymbol{r}, \boldsymbol{r}^{\prime}} & d_{\boldsymbol{r}, \boldsymbol{r}^{\prime}}^{\left(d_{\left.x^{2}-y^{2}\right)}\right.} \\
\left(d_{\boldsymbol{r}, \boldsymbol{r}^{\prime}}^{\left(d_{\left.x^{2}-y^{2}\right)}\right)^{*}}\right. & -h_{\boldsymbol{r}, \boldsymbol{r}^{\prime}}^{*}
\end{array}\right]\left[\begin{array}{l}
\left(u_{11}\right)_{\lambda}\left(\boldsymbol{r}^{\prime}\right) \\
\left(v_{21}\right)_{\lambda}\left(\boldsymbol{r}^{\prime}\right)
\end{array}\right]=E_{\lambda}\left[\begin{array}{l}
\left(u_{11}\right)_{\lambda}(\boldsymbol{r}) \\
\left(v_{21}\right)_{\lambda}(\boldsymbol{r})
\end{array}\right],
$$

where $u_{i j}\left(v_{i j}\right)$ represent elements of $\hat{u}(\hat{v})$ in Eq. (17). We omit 11 from $u_{11}$ and 21 from $v_{21}$ because $\left[u_{21}, v_{11}\right]^{t}$ obeys essentially the same equation as Eq. (20). The wave function at the $j$ th lattice sites in the $x$ direction can be represented in a column with $2 W$ elements:

$$
\boldsymbol{\Psi}_{\lambda}(j)=\left(\begin{array}{c}
u_{\lambda}(j \boldsymbol{x}+1 \boldsymbol{y}) \\
\vdots \\
u_{\lambda}(j \boldsymbol{x}+W \boldsymbol{y}) \\
v_{\lambda}(j \boldsymbol{x}+1 \boldsymbol{y}) \\
\vdots \\
v_{\lambda}(j \boldsymbol{x}+W \boldsymbol{y})
\end{array}\right) .
$$

For instance, for $j<-2$, the wave function obeys the BdG equation

$$
\begin{gathered}
\left(\begin{array}{cc}
-t \overline{1} & \frac{\Delta}{2} e^{i \varphi_{L}} \overline{1} \\
\frac{\Delta}{2} e^{-i \varphi_{L}} \overline{1} & t \overline{1}
\end{array}\right) \boldsymbol{\Psi}_{\lambda}(j+1) \\
+\left(\begin{array}{cc}
-E_{\lambda} \overline{1}+\bar{E}_{S} & \bar{T}_{S} \\
\bar{T}_{S}^{\dagger} & -E_{\lambda} \overline{1}-\bar{E}_{S}
\end{array}\right) \boldsymbol{\Psi}_{\lambda}(j) \\
+\left(\begin{array}{cc}
-t \overline{1} & \frac{\Delta}{2} e^{i \varphi_{L} \overline{1}} \\
\frac{\Delta}{2} e^{-i \varphi_{L} \overline{1}} & t \overline{1}
\end{array}\right) \boldsymbol{\Psi}_{\lambda}(j-1)=0 \\
\bar{T}_{S}=-\frac{\Delta}{2} e^{i \varphi_{L} \bar{h}_{0}},
\end{gathered}
$$

$$
\bar{E}_{S}=(-\mu+4 t) \overline{1}-t \bar{h}_{0},
$$




$$
\bar{h}_{0}=\left(\begin{array}{ccccc}
0 & 1 & 0 & \ldots & 1 \\
1 & 0 & 1 & \ldots & 0 \\
\vdots & \ddots & \ddots & \ddots & \vdots \\
0 & \cdots & 1 & 0 & 1 \\
1 & 0 & \cdots & 1 & 0
\end{array}\right),
$$

where $\bar{\cdots}$ denotes $W \times W$ matrices and $\overline{1}$ is the unit matrix. To solve the BdG equation, we apply the recursive Green function method ${ }^{32-34}$ and calculate the Matsubara Green function in a $2 W \times 2 W$ matrix form

$$
\check{G}_{\omega_{n}}\left(j, j^{\prime}\right)=\sum_{\lambda} \boldsymbol{\Psi}_{\lambda}(j)\left[i \omega_{n}-E_{\lambda}\right]^{-1} \boldsymbol{\Psi}_{\lambda}^{\dagger}\left(j^{\prime}\right) .
$$

The Josephson current in the normal metal $\left(1<j<L_{N}-1\right)$ is given by 32,33

$$
J(j)=-i e t T \sum_{\omega_{n}} \operatorname{Tr}\left[\check{G}_{\omega_{n}}(j+1, j)-\check{G}_{\omega_{n}}(j, j+1)\right] .
$$

We note that $J(j)$ is independent of $j$ when we consider the direct-current Josephson effect.

In simulations, we first compute the Josephson current for a single sample with a specific random potential configuration. After calculating the Josephson current over a number of samples with different random configurations, the ensemble average of the Josephson current and its fluctuations are obtained as

$$
\begin{gathered}
\langle J\rangle=\frac{1}{N_{s}} \sum_{i=1}^{N_{s}} J_{i}, \\
\delta J=\sqrt{\left\langle J^{2}\right\rangle-\langle J\rangle^{2}},
\end{gathered}
$$

where $J_{i}$ is the Josephson current in the $i$ th sample and $N_{s}$ is a number of samples. Strictly speaking, $N_{s}$ should be taken to be infinity. In this paper, we increase $N_{s}$ until a sufficient convergence of $\langle J\rangle$ and $\delta J$ is obtained.

\section{RESULTS}

\section{A. Ballistic junctions}

Before discussing the Josephson effect through diffusive normal metals, the Josephson current through ballistic metals should be briefly clarified. Throughout this paper, we fix the parameters as $L_{N}=70, W=25$, and $\mu=2.0 t$. In Fig. 2, we show current-phase relation $(\mathrm{CPR})$ for the $s^{-}, d_{x y^{-}}, p_{y^{-}}$, and $p_{x}$-wave symmetries, where $\Delta_{0}=0.01 t, T=0.001 T_{c}$, and $\varphi$ $=\varphi_{L}-\varphi_{R}$. Impurity potentials are not considered in normal metals (i.e., $V_{I}=0$ ), and Josephson currents are calculated for several choices of potential barriers $\left(V_{B}\right)$. In the absence of potential barriers for $V_{B}=0$, the CPR becomes $J \propto \varphi$ (Refs. 41 and 42) for all pairing symmetries. The ballistic junctions are rather close to the long limit because the coherence length $\left(\xi_{0}\right)$ estimated about at 50 lattice constant is slightly smaller than $L_{N} \cdot{ }^{41,42}$ This CPR is a direct consequence of the multiple Andreev reflection ${ }^{43}$ in low temperatures. In general, Josephson currents can be decomposed into a series of
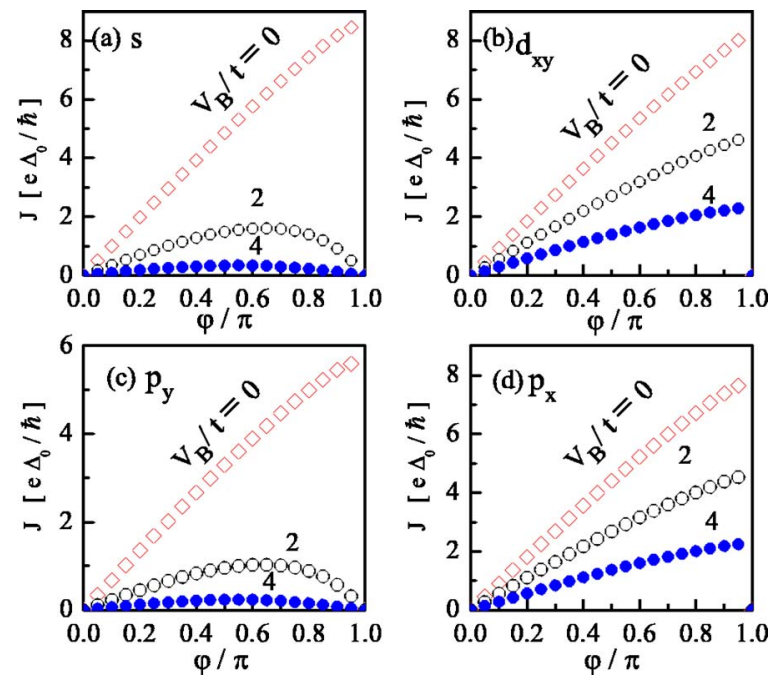

FIG. 2. (Color online) Current-phase relations in ballistic SNS junctions are shown for several choices of potential barriers $\left(V_{B}\right)$, where $T / T_{c}=0.001$ and $\Delta_{0} / t=0.01$. Impurity potentials are fixed at $V_{I}=0$.

$$
J=\sum_{n=1}^{\infty} J_{n} \sin (n \varphi) .
$$

The components of $J_{n}$ for $n \geq 2$ represent contributions of the multiple Andreev reflection. Roughly speaking, $J_{n}$ is proportional to $\left\{T_{N}\right\}^{n}$, where $T_{N}$ is the transmission probability of a quasiparticle from the left superconductor to the right superconductor through the normal segment (including two barriers and a normal metal). ${ }^{44}$ Thus the multiple Andreev reflection is negligible (i.e., $J_{1} \gg J_{2} \gg J_{3} \gg \cdots$ ) for $T_{N} \ll 1$. On the other hand, in the case of $T_{N}=1$, the multiple Andreev reflection leads to the deviation of current-phase relations from the sinusoidal function. It is noted at $T_{N}=1$ that we obtain $J$ $\propto \varphi$ and $J \propto \sin (\varphi / 2)$ at zero temperature for $L_{N} \gg \xi_{0}$ and $L_{N} \ll \xi_{0}$, respectively ${ }^{41,42,45}$ (see also Appendix B). The results of the CPR are close to the sinusoidal function as the increase of $V_{B}$ in the $s$ - and $p_{y}$-wave junctions because $T_{N}$ is suppressed by the potential barriers at $j=1$ and $L_{N}$ and contributions of the multiple Andreev reflection become small in the limit of large $V_{B}$. On the other hand, in the $d_{x y^{-}}$and $p_{x}$-wave symmetries, CPR's remain unchanged from $J \propto \varphi$ even in the presence of potential barriers. The multiple Andreev reflection is not suppressed in these symmetries because a transmission probability of insulating barriers is kept to unity due to the resonant tunneling through the MARS's.

In ballistic SNS junctions, the characteristic behavior of Josephson currents depends on the presence or absence of the MARS as shown in Fig. 1(b). We have confirmed that calculated results of the CPR in the $d_{x^{2}-y^{2} \text {-symmetry show }}$ qualitatively the same behavior as those in the $s$ - and $p_{y}$-wave symmetries.

\section{B. Diffusive junctions}

In Fig. 3, we show the CPR in disordered junctions at $V_{B}=2 t$, where $\Delta_{0}=0.01 t$ and $T=0.001 T_{c}$. At $V_{I}=2 t$, normal 

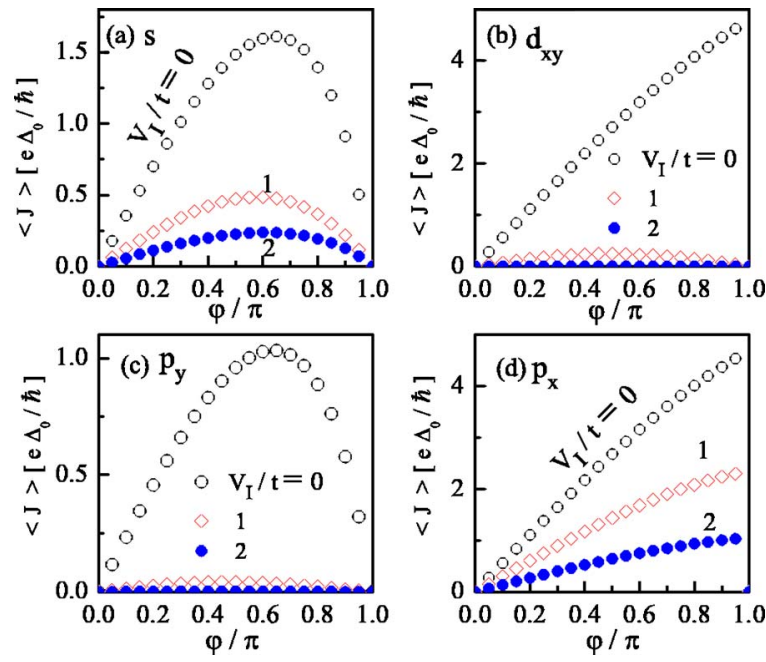

FIG. 3. (Color online) Current-phase relations in disordered SNS junctions are shown for several choices of impurity potentials $\left(V_{I}\right)$, where $T / T_{c}=0.001$ and $\Delta_{0} / t=0.01$. The barrier potential is fixed at $V_{B}=2 t$. At $V_{I}=2 t$, normal metals are in the diffusive transport regime.

metals are in the diffusive transport regime. The mean free path is about $\ell \sim 6$ lattice constants and the Thouless energy $E_{T h}$ is calculated to be $1.6 \times 10^{-3} t$. The amplitudes of Josephson currents decrease with the increase of $V_{I}$ in the $s$-wave junctions as shown in (a). Impurity potentials decrease the transmission probability of normal metals and therefore the amplitude of Josephson current. The ensemble average of Josephson currents for the $d_{x y}$-wave symmetry rapidly decreases with the increase of $V_{I}$ and vanishes in the diffusive limit. The same tendency can be seen in the $p_{y}$-wave symmetry. In the $d_{x y^{-}}$and $p_{y}$-wave symmetries, no Cooper pair exists in normal metals because the proximity effect is absent there.

In diffusive SNS junctions, it is reasonable to consider that the proximity effect governs the characteristic behavior of Josephson current. In the $d_{x y^{-}}$and $p_{y}$-wave symmetries, a relation $\langle J\rangle \sim 0$ is a natural consequence of the no proximity effect in diffusive metals. Thus it may be also reasonable to expect that the CPR in the $p_{x}$-wave symmetry would be close to the sinusoidal function in the presence of impurity potentials. The calculated results in (d), however, show that the CPR almost remains unchanged from $J \propto \varphi$ even in the diffusive limit. This implies that a quasiparticle is not scattered by impurity potentials at all. In the next subsection, we discuss the anomalous properties of Josephson current in the $p_{x}$-wave symmetry much further. Different from the $s$-wave symmetry, the MARS exists in the $p_{x}$-wave junctions as discussed in Fig. 1(b). The role of the MARS in the anomalous Josephson effect is explained in Sec. V.

Before turning to the anomalous Josephson effect in the $p_{x}$-wave symmetry, the fluctuations of Josephson current are clarified. In Fig. 4(a), we show the maximum amplitude of $\langle J\rangle$ and $\delta J$ for the $s$ - and $p_{x}$-wave symmetries are plotted, where $T_{B}$ is the transmission probability of potential barrier at interface in the normal state. Here we choose several values of the barrier potentials $V_{B}$ at $j=1$ and $L_{N}$. For instance,
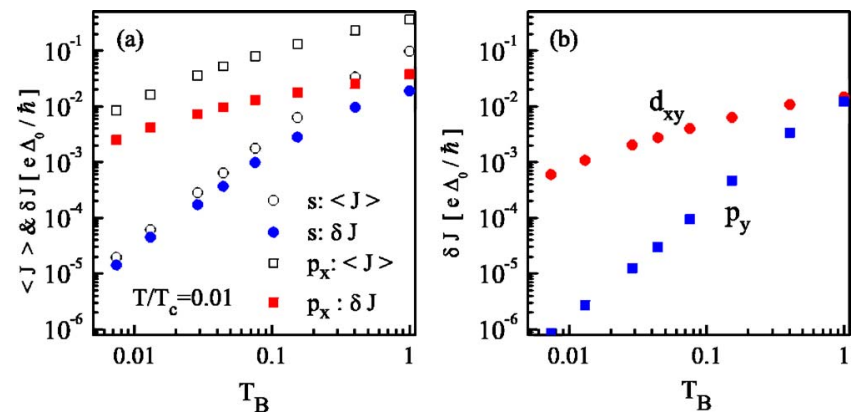

FIG. 4. (Color online) Ensemble average of Josephson current $\langle J\rangle$ and their fluctuations $\delta J$ are plotted as a function of transmission probability of potential barrier in the normal state $T_{B}$. Results for the $s$ - and $p_{x}$-wave symmetries are shown in (a). In (b), only $\delta J$ for the $d_{x y^{-}}$and $p_{y}$-wave symmetries are shown because $\langle J\rangle$ is much smaller than $\delta J$. Note that the scales of (a) and (b) are identical to each other.

the resulting normal transmission probabilities of the barrier $T_{B}$ are 1.0, 0.075, and 0.013 for $V_{B} / t=0,6$, and 15 , respectively. In the $s$-wave symmetry at $T_{B}=1, \delta J$ is close to analytical results of fluctuations. ${ }^{46,47}$ The amplitudes of fluctuations decrease with decreasing $T_{B}$ for both the $s$ - and $p_{x}$-wave junctions. For $T_{B} \ll 1,\langle J\rangle$ is close to $\delta J$ in the $s$-wave symmetry, whereas $\langle J\rangle$ is sufficiently larger than $\delta J$ in the $p_{x}$-wave symmetry. In (b), we show $\delta J$ for the $d_{x y^{-}}$and $p_{y}$-wave symmetries at $\varphi=\pi / 2$. Since the proximity effect is absent, we find a relation $|\langle J\rangle| \ll \delta J .{ }^{48}$ This relation does not mean the absence of Josephson current in a single sample measured in experiments. ${ }^{27}$ After calculating the Josephson current over a number of different samples, we find that about $50 \%$ of them are 0 junctions and the rest of them become $\pi$ junctions. As a consequence $|\langle J\rangle|$ becomes much smaller than $\delta J$. Thus Josephson current flows in a single sample although the direction of current depends on microscopic configurations of impurities. In Fig. 4(b), $\delta J$ represents the typical amplitude of Josephson current in a single sample. When we compare $\delta J$ in the $d_{x y}$-wave symmetry in (b) and $\langle J\rangle$ in the $s$-wave symmetry in (a), the amplitude of Josephson current in a single $d_{x y}$-wave junction is expected to be much larger than that in an $s$-wave junction in the limit of $T_{B} \ll 1$. This is because the MARS is forming at junction interfaces. ${ }^{26}$ In diffusive SNS junctions, the Josephson effect in the $d_{x y}$-wave symmetry is distinguished from that in the $p_{y}$-wave symmetry by the amplitude of fluctuations.

\section{Anomalous Josephson effect in $p_{x}$-wave symmetry}

At first we show that the maximum amplitudes of Josephson currents in the $p_{x}$-wave symmetry $J_{c}\left(p_{x}\right)$ become much larger than those in the $s$-wave symmetry $J_{c}(s)$. In Fig. 5(a), the ratios $J_{c}\left(p_{x}\right) / J_{c}(s)$ at $\Delta_{0}=0.1 t$ are plotted as a function of temperature for several choices of $T_{B}$. The ratios $J_{c}\left(p_{x}\right) / J_{c}(s)$ increase with decreasing $T$ and amazingly become more than 100 in low temperatures for small $T_{B}$. The amplitudes of Josephson currents in the $p_{x}$-wave junctions are much larger than those in the $s$-wave junctions. In Fig. 5(b), $J_{c} R_{N}$ normalized by $\pi \Delta_{0} / e$ is plotted as a function of $T_{B}$ at $T=0.001 T_{c}$, 

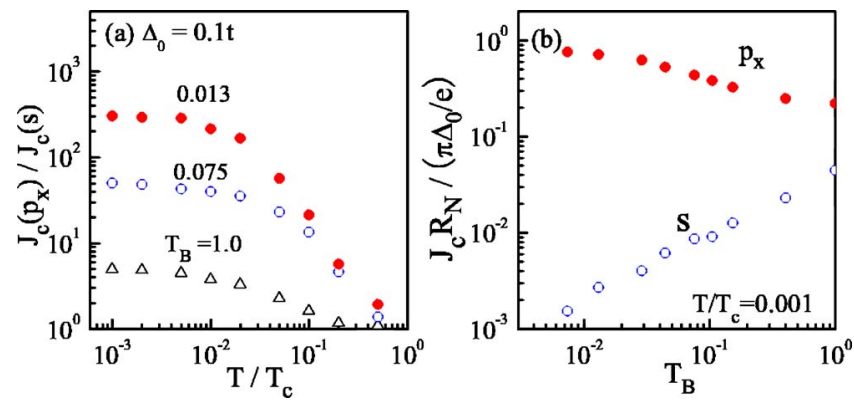

FIG. 5. (Color online) The maximum amplitudes of Josephson currents in the $p_{x}$-wave symmetry $J_{c}\left(p_{x}\right)$ are compared with those in the $s$-wave symmetry $J_{c}(s)$ in (a), where $T_{B}$ is the transmission probability of potential barriers in the normal states. In (b), $J_{c}\left(p_{x}\right)$ and $J_{c}(s)$ are plotted as a function of $T_{B}$ at $T=0.001 T_{c}$.

where $R_{N}$ is the normal resistance of junctions. The results show that $J_{c} R_{N}$ in the $s$-wave symmetry decreases with decreasing $T_{B}$, whereas that in the $p_{x}$-wave symmetry increases. It is known that large values of $J_{c}$ are desired in device applications because $J_{c} R_{N}$ limits the operation speeds of Josephson devices. Thus Fig. 5(b) implies high potentials of $p$-wave junctions as coherent devices.

We next focus on the current-phase relations of the Josephson effect. In Fig. 6, Josephson currents are plotted as a function of $\varphi$ for the $p_{x}$-wave symmetries at $V_{B}=0$. Parameters are chosen as $\Delta_{0}=0.01 t$ and $0.0001 t$ in (a) and (b), respectively. The CPR's are almost a sinusoidal function at a high temperature of $T=0.5 T_{c}$. At $T=0.001 T_{c}$, however, the current-phase relations are close to $J \propto \varphi$ and $J \propto \sin (\varphi / 2)$ in (a) and (b), respectively. These are characteristic currentphase relations in ballistic Josephson junctions in the $s$-wave symmetry. ${ }^{41,42,45}$ The results imply large contributions of the multiple Andreev reflection in low temperatures.

In Fig. 6, we also show the current-phase relations in the $s$-wave symmetry at $T=0.001 T_{c}$ with a solid line. The current-phase relation in the $s$-wave symmetry is described almost by the sinusoidal function ${ }^{4,49}$ because impurity potentials in normal metals suppress $T_{N}$ (transmission probability of the normal segment in a superconducting state) and therefore multiple Andreev reflections. In the $p_{x}$-wave junctions,
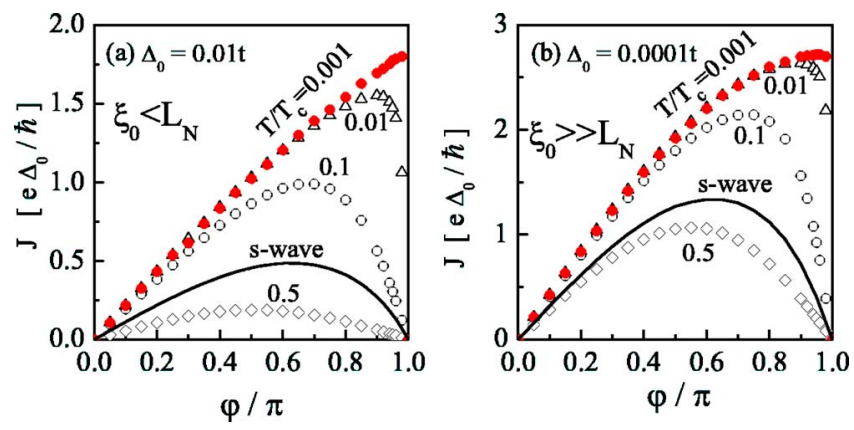

FIG. 6. (Color online) Current-phase relations for the $p_{x}$-wave symmetry are shown for several temperatures at $V_{B}=0$, where $\Delta_{0}$ $=0.01 t$ in (a) and $\Delta_{0}=0.0001 t$ in (b). For comparison, results in the $s$-wave junctions at $T=0.001 T_{c}$ are shown with a solid line in (a) and (b). The amplitude in the $s$-wave symmetry is multiplied by 5 in (a). the coherence length $\xi_{0}$ is estimated to be about 50 lattice constants in (a) and 5000 in (b). Thus $L_{N}>\xi_{0}$ and $L_{N} \ll \xi_{0}$ are satisfied in (a) and (b), respectively. The current-phase relations such as $J \propto \sin (\varphi / 2)$ in (b) and $J \propto \varphi$ in (a) are universal properties of the $p_{x}$-wave junctions at low temperatures because they are independent of the strength of the barrier potentials and the degree of disorder in normal metals. The calculated results in Fig. 6 indicate $T_{N}=1$ even in the presence of impurity potentials. The large amplitudes of the Josephson current in Fig. 5 are also explained by $T_{N}=1$.

\section{MARS IN NORMAL METALS}

\section{A. Quasiparticle density of states}

The calculated results in Figs. 3(d), 5, and 6 show the specific properties of Josephson currents in the $p_{x}$-wave junctions. In what follows, we analyze quasiparticle states in normal metals to understand the origin of the anomalous Josephson effect. In Fig. 7, we show the local density of states in normal metals for the $s$ - and $p_{x}$-wave symmetries, where $\Delta_{0}=0.005 t, \gamma=0.05 \Delta_{0}$, and $N_{0}$ denotes the normal density of states. The local density of states is calculated from

$$
N(E, j)=-\operatorname{Im} \operatorname{Tr} \check{G}_{E+i \gamma}(j, j) / \pi,
$$

where $E$ is measured from the Fermi energy and $\gamma$ is a small imaginary part. At $\varphi=0$ in the $s$-wave junctions in (a), the local density of states for $E<E_{T h} \sim 0.3 \Delta_{0}$ is suppressed because of the proximity effect. The suppression of the local density of states indicates the conversion of quasiparticles to Cooper pairs in normal metals. At $\varphi=\pi$ in (b), the local density of states recovers its amplitude for $E<E_{T h}$. The wave functions of Cooper pairs from the left superconductor and from the right one cancel each other around $\varphi \sim \pi$ as schematically illustrated in Fig. 8.

The local density of states is drastically changed in the $p_{x}$-wave symmetry as shown in Fig. 8(c) and 8(d). Zeroenergy peaks whose width is determined by $\gamma$ can be seen, which means the formation of the midgap Andreev resonant state in normal metals. Although the MARS originally localizes at junction interfaces, ${ }^{11}$ the MARS penetrates into normal metals in the presence of the proximity effect. Spatial profiles of the local density of states depend remarkably on the external phase difference as shown in Figs. 8(c) and 8(d). At $\varphi=0$, the zero-energy peak disappears at the center of normal metals $(j \sim 35)$ because the wave functions of the MARS from the left superconductors cancel out that from the right one as shown schematically in a lower panel in Fig. 8(c). On the other hand, in Fig. 8(d), the wave functions of the MARS in the two superconductors have the same sign with each other. Thus the two MARS's can penetrate deeply into normal metals and the zero-energy peak can be seen everywhere. We note that the penetration of the MARS is possible only when the proximity effect is present in normal metals. In fact, we have confirmed that no zero-energy peak is found in normal metals in the $d_{x y}$-wave symmetry as shown in Fig. 8(a), where the LDOS for $d_{x y}$-wave and $p_{y}$-wave symmetries are plotted for $j=0-71$ in Figs. 8(a) and 8 (b), respectively. In normal metals for $j=1 \sim 70$, the LDOS 


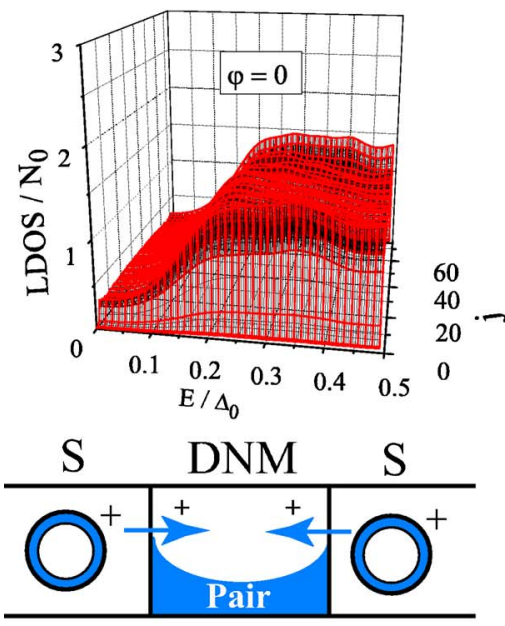

(a) s-wave: $\varphi=0$
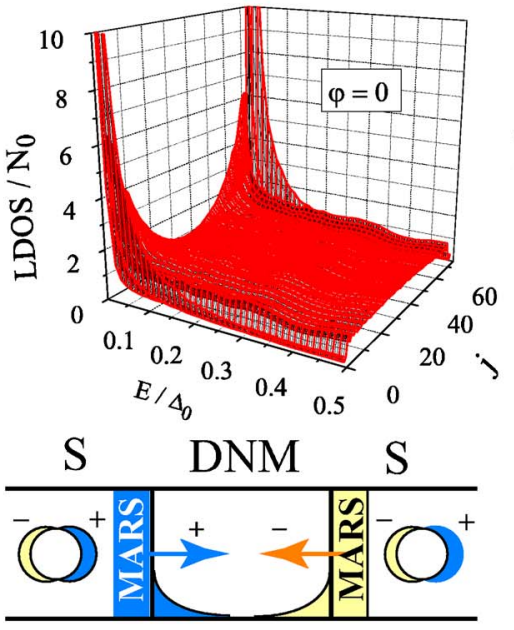

(c) $p_{\mathrm{x}}$-wave: $\varphi=0$
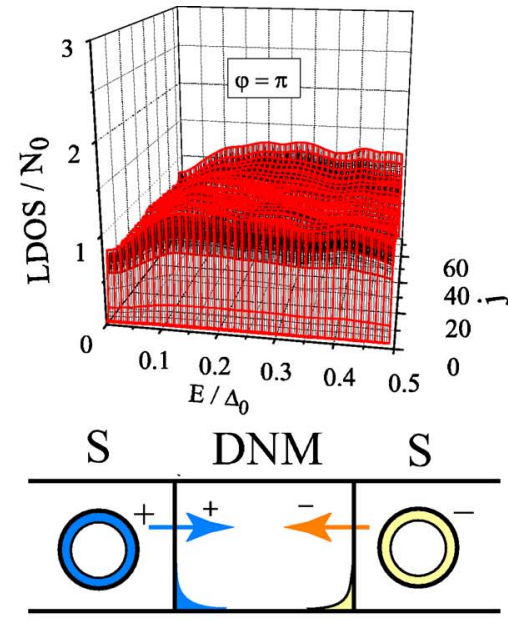

(b) s-wave: $\varphi=\pi$
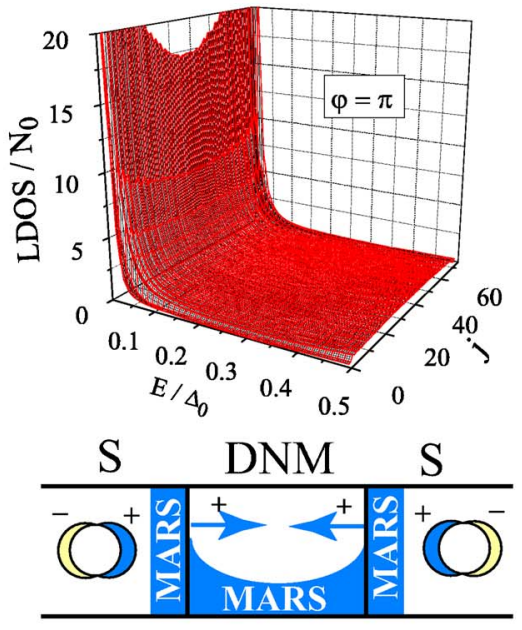

(d) $p_{x}$-wave: $\varphi=\pi$
FIG. 7. (Color online) Local density of states (LDOS) in normal metals $\left(0 \leq j \leq L_{N}=71\right)$ is shown for the $s$-wave and $p_{x}$-wave symmetries. The left and right superconductors are attached at $j=0$ and $j=71$, respectively. Note that $E_{T h}$ is about $0.3 \Delta_{0}$. In schematic pictures, DNM and $S$ denote a diffusive normal metal and a superconductor, respectively. The local density of states shown here is calculated in the absence of Josephson currents. We have confirmed that the results at $\varphi=0.99 \pi$ qualitatively show the same behavior as those at $\varphi=\pi$. becomes almost a flat structure around $E \sim 0$ in both $d_{x y^{-}}$and $p_{y}$-wave symmetries because the proximity effect is absent there. In the $d_{x y}$-wave symmetry, zero-energy peaks in LDOS at $j=0$ and 71 indicate the formation of the MARS in superconductors and are responsible for the large fluctuations shown in Fig. 4(b). Figure 7 indicates that the proximity effects bridges the two MARS's in the two superconductors.

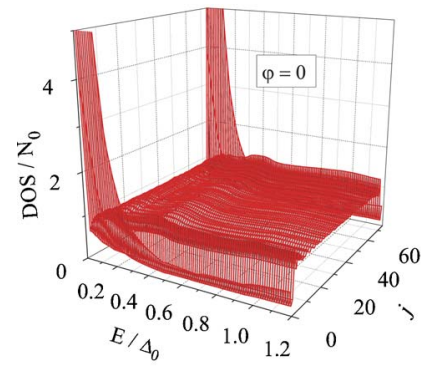

(a) $d_{x y}$ - wave

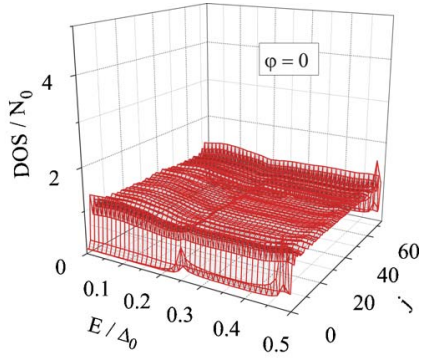

(b) $\mathrm{p}_{\mathrm{y}}$ - wave
FIG. 8. (Color online) Local density of states at $\varphi=0$ in normal metals are shown for the $d_{x y}$-wave and $p_{y}$-wave symmetries in (a) and (b), respectively. In the $d_{x y}$ symmetry, zero-energy peaks at $j=0$ and 71 correspond to the MARS.
Thus $T_{N}=1$ holds because of the resonant transmission through the MARS in normal metals. The Josephson effect specific to the $p_{x}$-wave symmetry discussed in Figs. 5 and 6 are a consequence of the diffusion of the MARS into normal metals. ${ }^{50}$

\section{B. Cooper pairs in diffusive normal metals}

In normal metals, pair potentials are always zero because of no pairing interaction. The proximity effect means finite Cooper pair amplitudes in normal metals. When the Green function in Eq. (26) is expressed by

$$
\check{G}_{\omega_{n}}\left(j, j^{\prime}\right)=\left(\begin{array}{ll}
\bar{a}_{\omega_{n}}\left(j, j^{\prime}\right) & \bar{b}_{\omega_{n}}\left(j, j^{\prime}\right) \\
\bar{c}_{\omega_{n}}\left(j, j^{\prime}\right) & \bar{d}_{\omega_{n}}\left(j, j^{\prime}\right)
\end{array}\right),
$$

pair amplitudes at $j$ are calculated to be $\operatorname{Tr} \bar{b}_{\omega_{n}}(j, j)$. In superconductors, the pair amplitude is an even function of $\omega_{n}$ irrespective of pairing symmetries. In the $s$-wave symmetry, the pair amplitude in a diffusive normal metal is also an even function of $\omega_{n}$. In the $p_{x}$-wave symmetry, however, we confirmed that the pair amplitude becomes an odd function of $\omega_{n}$ 
in a diffusive normal metal. Thus odd-frequency pairs carry the anomalous Josephson current. This is a consequence of Pauli's principle in quantum mechanics. A general theory of the odd-frequency pairs in proximity structures will be given elsewhere..$^{51}$

\section{CONCLUSION}

We have numerically studied the Josephson current in superconductor/diffusive-normal-metal/superconductor junctions by using the recursive Green function method. In superconductors, we assume $s-, d_{x y^{-}}, p_{y^{-}}$, and $p_{x^{-}}$wave pairing symmetries. These pair potentials are classified into different groups by the presence or absence of two interference effects: a midgap Andreev resonant state at junction interfaces and the proximity effect in diffusive normal metals as shown in Fig. 1(b). In the $d_{x y^{-}}$and $p_{y^{-}}$-wave symmetries, the ensemble average of the Josephson current vanishes (i.e., $|\langle J\rangle| \ll \delta J)$ for all temperatures because the proximity effect is absent in diffusive normal metals.

The Josephson current in the $p_{x}$-wave symmetry is much larger than that in the $s$-wave in low temperatures. In the $p_{x}$-wave symmetry, current-phase relations in low temperatures are close to those in ballistic junctions such as $J \propto \sin (\varphi / 2)$ and $J \propto \varphi$ independent of the strength of potential barriers at interfaces and the degree of disorder in normal metals. This is a consequence of a cooperative effect between the MARS at junction interfaces and the proximity effect in diffusive normal metals. The two midgap Andreev resonant states penetrate deeply into normal metals and causes the unusual Josephson effect in the $p_{x}$-wave superconducting junctions. The anomalous Josephson effect is a novel feature of phase-sensitive transport in spin-triplet superconducting junctions.

\section{ACKNOWLEDGMENTS}

This work has been partially supported by a Grant-in-Aid for the 21st Century COE program on "Topological Science and Technology" and "Frontiers of Computational Science" and a Grant-in-Aid for Scientific Research on Priority Area "Physics of new quantum phases in superclean materials" (Grant No. 18043001) from The Ministry of Education, Culture, Sports, Science and Technology of Japan.

\section{APPENDIX A: NORMAL CONDUCTANCE}

In this appendix, the normal conductance in disordered wires is summarized. We fix two parameters such as $V_{I}$ $=2.0 t$ and $\mu=2.0 t$. In Fig. 9, we show the conductance of the normal segment at zero temperature as a function of $L_{N}$ by using the usual recursive Green function method. ${ }^{34}$ When the normal metals are in the quasi ballistic regime, $\left\langle g_{N}\right\rangle\left(L_{N}\right) / W$ is proportional to $L_{N}$ because $\left\langle g_{N}\right\rangle \propto N_{c}$. Here $N_{c}$ is the number of propagating channels, which is almost proportional to $W$. When the normal metals are in the diffusive transport regime, $\left\langle g_{N}\right\rangle\left(L_{N}\right) / W$ becomes $k_{F} \ell / 2$ independent of $L_{N}$, where $\ell$ is the elastic mean free path. When the normal metals are in the localization regime, $\left\langle g_{N}\right\rangle L_{N} / W$ decreases with

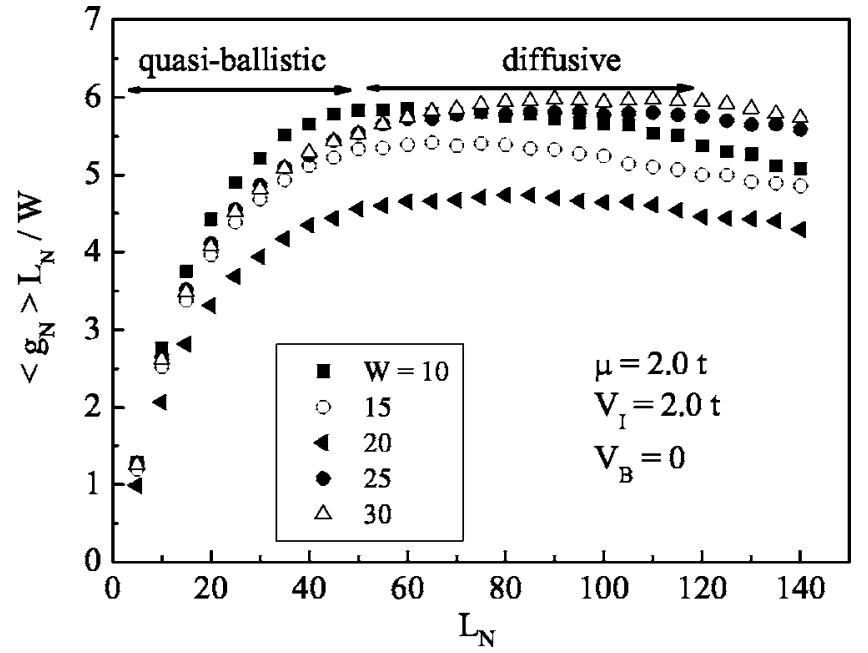

FIG. 9. The normal conductance versus the length of disordered region is plotted, where $V_{I}=2.0 t, \mu=2.0 t$, and $V_{B}=0$.

increasing $L_{N}$ like $\exp \left(-L_{N} / \xi_{L}\right)$, where $\xi_{L}$ is the localization length. Thus, in Fig. 9, the quasiballistic, the diffusive, and the localization regimes correspond to $L_{N}<50,60<L_{N}$ $<110$, and $L_{N} \gg 110$, respectively. In the diffusive regime, all data in (a) should be close to a single curve irrespective of $W$. The results, however, slightly depend on $W$ because of finite-size effects. Strictly speaking, $N_{c}$ is not exactly proportional to $W$. From the numerical results for $W=25$ in the diffusive regime, we estimate the mean free path as $\ell \sim 6$ lattice constants. When we fix $L_{N}$ at 70, the Thouless energy $E_{T h}$ is estimated to be $1.6 \times 10^{-3} t$.

\section{APPENDIX B: CURRENT-PHASE RELATION AT $T=0$}

The relation between the pairing symmetries and the proximity effect can be understood by an analytical expression of the Josephson current. ${ }^{19,26,27}$ The Josephson current is decomposed into a series as shown in Eq. (30). The components of Josephson current can be represented approximately by a formula ${ }^{19}$

$$
\begin{aligned}
J_{n} \sin (n \varphi)= & -2 e \operatorname{Im} \sum_{k_{y}}{ }^{\prime} \sum_{k_{y}}{ }^{\prime} T \sum_{\omega_{n}} \operatorname{Tr} \\
& \times\left[\left(\hat{r}^{e h}\left(k_{y}\right) \cdot \hat{t}_{k_{y}, k_{y}^{\prime}} \cdot \hat{r}^{h e}\left(k_{y}^{\prime}\right) \cdot \hat{t}_{k_{y}^{\prime}, k_{y}}^{e}\right)^{n}\right],
\end{aligned}
$$

where $\hat{r}^{e h}\left(k_{y}\right)\left[\hat{r}^{h e}\left(k_{y}^{\prime}\right)\right]$ is the Andreev reflection coefficient ${ }^{43}$ from a hole to an electron at the left interface [from an electron to a hole at the right interface] and $\hat{t}^{e}\left[\hat{t}^{h}\right]$ is the transmission coefficient in a diffusive normal metal in the electron [hole] branch. The propagation process is schematically illustrated in Fig. 1(c). At the left (right) interface, $k_{y}\left(k_{y}^{\prime}\right)$ characterizes a transverse momentum (a transport channel) on the Fermi surface. The summation $\Sigma_{k_{y}}^{\prime}$ runs over wave numbers $k_{y}$ on the Fermi surface with $k_{x}>0$.

When we consider a $\delta$-function potential barrier at the interface, $V(\boldsymbol{r})=V_{0} \delta(\boldsymbol{r})$, we obtain expressions of the Andreev reflection coefficients ${ }^{19}$ 


$$
\begin{gathered}
\hat{r}^{e h}\left(k_{y}\right)=-i \vec{k}_{x}^{2} \frac{t_{B} K}{\Xi} \nu \hat{\Delta}_{k} e^{i \varphi_{L},} \\
\hat{r}^{h e}\left(k_{y}\right)=-i\left[\frac{t_{B} K}{\Xi} \nu \hat{\Delta}_{k}\right]^{\dagger} e^{-i \varphi_{R},} \\
K=\sqrt{\left(\omega_{n}\right)^{2}+\Delta_{k}^{2}}-\left|\omega_{n}\right|, \\
\Xi=\Delta_{k}^{2} \nu+\left(1-t_{B}\right) K^{2}, \\
\hat{\Delta}_{k}=\left\{\begin{array}{cc}
i \Delta_{k} \hat{\sigma}_{2}: & \text { singlet, } \\
i \Delta_{k}(\boldsymbol{e} \cdot \hat{\boldsymbol{\sigma}}) \hat{\sigma}_{2}: & \text { triplet, }
\end{array}\right.
\end{gathered}
$$

where $t_{B}=\vec{k}_{x}^{2} /\left(z_{0}^{2}+\vec{k}_{x}^{2}\right)$ is the transmission probability of the barrier for a wave number $\left(\bar{k}_{x}, \bar{k}_{y}\right), z_{0} \equiv V_{0} / \hbar v_{F}$ represents the strength of the potential barrier, $\hat{\sigma}_{j}$ with $j=1-3$ are the Pauli matrices, and $\Delta_{k}$ is given in Eqs. (1)-(5). Here we assume that $\Delta_{-k_{x}, k_{y}}=\nu \Delta_{k_{x}, k_{y}}$ with $\nu= \pm 1$. The pair potentials in Eqs. (1), (2), and (4) belong to $\nu=1$, whereas Eqs. (3) and (5) belong to $\nu=-1$. The spin part of Cooper pairs is taken into account through a matrix representation of the pair potential in Eq. (B6).The transmission coefficients in normal metals are spin diagonal (i.e., $\hat{t}^{e(h)}=t^{e(h)} \hat{\sigma}_{0}$ ) in the absence of spin-flip scatterings, where $\hat{\sigma}_{0}$ is the $2 \times 2$ unit matrix. The ensemble average of $t_{k_{y}^{\prime}, k_{y}}^{e} t_{k_{y}, k_{y}^{\prime}}^{h}$ can be represented by the Cooperon propagator because the propagation paths of a hole are the time-reversal propagation paths of an electron as shown in Fig. 1(c). In diffusive normal metals, the transmission coefficients after the ensemble average are calculated as

$$
\begin{gathered}
\left\langle\left( t_{k_{y}^{\prime}, k_{y}}^{e} t_{\left.\left.k_{y}, k_{y}^{\prime}\right)^{n}\right\rangle}^{h} \simeq \frac{1}{N_{c}^{2}}\left\langle g_{N}\left(n L_{N}\right)\right\rangle \frac{\eta\left(n L_{N}\right)}{\sinh \eta\left(n L_{N}\right)},\right.\right. \\
\eta\left(L_{N}\right)=\sqrt{\frac{\omega_{n}}{\pi T}} \frac{L_{N}}{\xi_{D}}=\sqrt{\frac{2 \omega_{n}}{E_{T h}}}, \\
\left\langle g_{N}\left(L_{N}\right)\right\rangle=\frac{W}{L_{N}} \pi N_{0} D_{0},
\end{gathered}
$$

where $\langle\cdots\rangle$ denotes the ensemble average, $N_{c}=\left(W k_{F} / \pi\right)$ is the number of propagating channels, $N_{0}$ is the density of states per unit area, and $W$ is the width of junctions. In diffusive normal metals, $\xi_{D}=\sqrt{D_{0} / 2 \pi T}$ is the coherence length and $\left\langle G_{N}\right\rangle=\left(2 e^{2} / h\right)\left\langle g_{N}\right\rangle$ is the ensemble average of the normal conductance. After the ensemble average, the transmission coefficients are independent of $k_{y}$ and $k_{y}^{\prime}$. We note that $\eta / \sinh \eta$ represents a degree of phase coherence in normal metals. The phase coherence in normal metals is perfect (i.e., $\eta / \sinh \eta=1)$ at zero temperature. The phase coherence is suppressed in high temperatures such as $\omega_{n} \gg E_{T h}$.

After a small amount of algebra, we find

$$
\left\langle J_{1}\right\rangle=4 e T \sum_{\omega_{n}}\left\langle g_{N}\right\rangle \frac{\eta}{\sinh \eta} I_{1}^{2}
$$

$$
I_{n}=\frac{1}{N_{c}} \sum_{k_{y}},\left\{\frac{t_{B} K \nu}{\Xi} \Delta_{k}\right\}^{n}
$$

where $I_{1}$ corresponds to the Andreev reflection coefficient averaged over all propagating channels. We find $I_{1}=0$ for the $d_{x y^{-}}$and $p_{y}$-wave symmetries because $\vec{k}_{x}^{2} K / \Xi$ is an even function of $k_{y}$ and $\Delta_{k}$ is an odd function of $k_{y}$. Since $\left\langle J_{1}\right\rangle$ $\propto I_{1}^{2}$, the ensemble average of $J_{1}$ vanishes, which represents the absence of the proximity effect. Even in unconventional superconductor junctions, Cooper pairs penetrate into normal metals. However, Cooper pairs in normal metals are characterized not only by the amplitude but also by the sign degree of freedom. When Eq. (7) holds, Cooper pairs with positive sign and those with negative sign cancel each other out in diffusive normal metals.

In the presence of the MARS, the expansion in Eq. (B1) has less validity. The effects of the MARS on the Josephson current, however, can be roughly understood with the following argument. In the limit of $\omega_{n} \rightarrow 0$ and $t_{B} \ll 1$, we find

$$
\frac{t_{B} K \nu \Delta_{k}}{\Xi} \rightarrow\left\{\begin{array}{lc}
\frac{t_{B}}{2} \frac{\Delta_{k}}{\sqrt{\omega_{n}^{2}+\Delta_{k}^{2}}}, & \nu=1, \\
\frac{t_{B} \Delta_{k}}{2\left|\omega_{n}\right|+t_{B}\left|\Delta_{k}\right|}, & \nu=-1 .
\end{array}\right.
$$

For $\nu=-1$, the Andreev reflection coefficient becomes unity independent of $t_{B}$ in the limit of $\omega_{n} \rightarrow 0$. The Josephson current in the presence of the MARS is expected to be anomalous at low temperatures because of the rapid increase of $I_{1}$ with the decrease of $\omega_{n} \propto T$.

In the case of $s$-wave symmetry, the characteristic CPR at the zero temperature can be derived from Eq. (B1). In ballistic junctions, transmission coefficients are approximately given by

$$
t_{k_{y}, k_{y}^{\prime}}^{h} t_{k_{y}^{\prime}, k_{y}}^{e}=\delta_{k_{y}^{\prime}, k_{y}} \exp \left(-2 \omega_{n} L_{N} / v_{F}\right) .
$$

At $T=0, t_{k_{y}, k_{y}^{\prime}}^{h} t_{k_{y}^{\prime}, k_{y}}^{e}$ corresponds to the transmission probability in the normal segment and becomes unity (i.e., $T_{N}=1$ ). Thus we obtain, at $z_{0}=0$,

$$
J_{n}=4 e(-1)^{n+1} T \sum_{\omega_{n}} \sum_{k_{y}}{ }^{\prime} e^{-2 \omega_{n} L_{N} n v_{F}} \times\left(\frac{\sqrt{\omega_{n}^{2}+\Delta_{0}^{2}}-\left|\omega_{n}\right|}{\Delta_{0}}\right)^{2 n} .
$$

The summation of $\omega_{n}$ can be replaced by the integration at $T=0$. We obtain

$$
J_{n}= \begin{cases}N_{c} \frac{2(-1)^{n+1}}{n} \frac{e v_{F}}{\pi L_{N}} & \text { for } \xi_{0} \ll L_{N}, \\ N_{c} \frac{8(-1)^{n+1}}{(2 n+1)(2 n-1) \pi} e \Delta_{0} & \text { for } \xi_{0} \gg L_{N} .\end{cases}
$$

The CPR are calculated as ${ }^{41,42,45}$ 


$$
J= \begin{cases}N_{c} \frac{e v_{F}}{L_{N}} \frac{\varphi}{\pi} & \text { for } \xi_{0} \ll L_{N}, \\ N_{c} e \Delta_{0} \sin (\varphi / 2) & \text { for } \xi_{0} \gg L_{N} .\end{cases}
$$

When normal metals are in the diffusive limit,

$$
\begin{aligned}
J_{n}= & 4 e(-1)^{n+1} T \sum_{\omega_{n}} \frac{\left\langle g_{N}\left(L_{N}\right)\right\rangle}{n} e^{-n \sqrt{2 \omega_{n} / E_{T h}}} \\
& \times\left(\frac{\sqrt{\omega_{n}^{2}+\Delta_{0}^{2}}-\left|\omega_{n}\right|}{\Delta_{0}}\right)^{2 n}
\end{aligned}
$$

is obtained from Eqs. (B7)-(B9) at $z_{0}=0$. For $\Delta_{0} \ll E_{T h}$, we find

$$
J_{n}=4 e\left\langle g_{N}\left(L_{N}\right)\right\rangle \frac{\Delta_{0}}{\pi} \frac{2(-1)^{n+1}}{(2 n+1)(2 n-1)} .
$$

The CPR

$$
J=\frac{4}{\pi} e \Delta_{0}\left\langle g_{N}\left(L_{N}\right)\right\rangle \cos (\varphi / 2) a \tanh [\sin (\varphi / 2)]
$$

coincides with the previous results ${ }^{49}$ apart from a numerical factor. In the same way, we obtain

$$
J=e E_{T h} \frac{4}{\pi}\left\langle g_{N}\left(L_{N}\right)\right\rangle \sum_{n=1}^{\infty} \frac{(-1)^{n+1}}{n^{3}} \sin (n \varphi)
$$

for $\Delta_{0} \gg E_{T h}$. The results, however, do not coincide with those in Ref. 4. Equation (B20) takes its maximum at $\varphi=0.58 \pi$. On the other hand, the results in Ref. 4 show the maximum around $\varphi=0.62 \pi$ consistently with numerical simulations.
*Electronic address: asano@eng.hokudai.ac.jp

${ }^{1}$ B. D. Josephson, Phys. Lett. 1, 251 (1962).

${ }^{2}$ K. K. Likharev, Rev. Mod. Phys. 51, 101 (1979).

${ }^{3}$ V. Ambegaokar and A. Baratoff, Phys. Rev. Lett. 10, 486 (1963).

${ }^{4}$ A. D. Zaikin and G. F. Zharkov, Sov. J. Low Temp. Phys. 7, 184 (1981)

${ }^{5}$ C. C. Tsuei and J. R. Kirtley, Rev. Mod. Phys. 72, 969 (2000).

${ }^{6}$ M. Sigrist and T. M. Rice, J. Phys. Soc. Jpn. 61, 4283 (1992); Rev. Mod. Phys. 67, 503 (1995).

${ }^{7}$ D. A. Wollman, D. J. Van Harlingen, W. C. Lee, D. M. Ginsberg, and A. J. Leggett, Phys. Rev. Lett. 71, 2134 (1993).

${ }^{8}$ L. J. Buchholtz and G. Zwicknagl, Phys. Rev. B 23, 5788 (1981).

${ }^{9}$ J. Hara and K. Nagai, Prog. Theor. Phys. 76, 1237 (1986).

${ }^{10}$ C. R. Hu, Phys. Rev. Lett. 72, 1526 (1994).

${ }^{11}$ Y. Tanaka and S. Kashiwaya, Phys. Rev. Lett. 74, 3451 (1995).

${ }^{12}$ S. Kashiwaya and Y. Tanaka, Rep. Prog. Phys. 63, 1641 (2001).

${ }^{13}$ Y. Asano, Y. Tanaka, and S. Kashiwaya, Phys. Rev. B 69, 134501 (2004).

${ }^{14}$ Y. Asano, Y. Tanaka, and S. Kashiwaya, Phys. Rev. B 69, 214509 (2004).

${ }^{15}$ Y. Tanaka and S. Kashiwaya, Phys. Rev. B 53, R11957 (1996).

${ }^{16}$ Y. S. Barash, H. Burkhardt, and D. Rainer, Phys. Rev. Lett. 77, 4070 (1996).

${ }^{17}$ Y. Tanaka and S. Kashiwaya, Phys. Rev. B 56, 892 (1997).

${ }^{18}$ Y. Tanaka and S. Kashiwaya, Phys. Rev. B 58, R2948 (1998).

${ }^{19}$ Y. Asano, Phys. Rev. B 64, 224515 (2001).

${ }^{20}$ Y. S. Barash, A. M. Bobkov, and M. Fogelström, Phys. Rev. B 64, 214503 (2001).

${ }^{21}$ Y. Asano and K. Katabuchi, J. Phys. Soc. Jpn. 71, 1974 (2002).

${ }^{22}$ Y. Asano, Y. Tanaka, M. Sigrist, and S. Kashiwaya, Phys. Rev. B 67, 184505 (2003); 71, 214501 (2005).

${ }^{23}$ E. Il'ichev, V. Zakosarenko, R. P. J. Ijsselsteijn, V. Schultze, H.-G. Meyer, H. E. Hoenig, H. Hilgenkamp, and J. Mannhart, Phys. Rev. Lett. 81, 894 (1998); E. Il'ichev, M. Grajcar, R. Hlubina, R. P. J. Ijsselsteijn, H. E. Hoenig, H. -G. Meyer, A. Golubov, M. H. S. Amin, A. M. Zagoskin, A. N. Omelyanchouk, and M. Yu. Kupriyanov, ibid. 86, 5369 (2001).
${ }^{24}$ H.-J. Kwon, K. Senguputa, and V. M. Yakovenko, Eur. Phys. J. B 37, 349 (2004).

${ }^{25}$ A. A. Golubov, M. Yu. Kupriyanov, and E. Il'ichev, Rev. Mod. Phys. 76, 411 (2004).

${ }^{26}$ Y. Asano, Phys. Rev. B 64, 014511 (2001).

${ }^{27}$ Y. Asano, J. Phys. Soc. Jpn. 71, 905 (2002).

${ }^{28}$ Y. Tanaka, Yu. V. Nazarov, and S. Kashiwaya, Phys. Rev. Lett. 90, 167003 (2003).

${ }^{29}$ Y. Tanaka, Yu. V. Nazarov, A. A. Golubov, and S. Kashiwaya, Phys. Rev. B 69, 144519 (2004).

${ }^{30}$ Y. Tanaka and S. Kashiwaya, Phys. Rev. B 70, 012507 (2004).

${ }^{31}$ Y. Tanaka, S. Kashiwaya, and T. Yokoyama, Phys. Rev. B 71, 094513 (2005); Y. Tanaka, Y. Asano, A. A. Golubov, and S. Kashiwaya, Phys. Rev. B 72, 140503(R) (2005).

${ }^{32}$ A. Furusaki, Physica B 203, 214 (1994).

${ }^{33}$ Y. Asano, Phys. Rev. B 63, 052512 (2001).

${ }^{34}$ P. A. Lee and D. S. Fisher, Phys. Rev. Lett. 47, 882 (1981).

${ }^{35}$ Y. Asano, Y. Tanaka, and S. Kashiwaya, Phys. Rev. Lett. 96, 097007 (2006)

${ }^{36}$ A. A. Abrikosov, J. Low Temp. Phys. 53, 359 (1983).

${ }^{37}$ Y. Hasegawa and H. Fukuyama, J. Phys. Soc. Jpn. 56, 877 (1987).

${ }^{38}$ A. G. Lebed, Phys. Rev. B 59, R721 (1999); A. G. Lebed, K. Machida, and M. Ozaki, Phys. Rev. B 62, R795 (2000).

${ }^{39}$ Y. Maeno, H. Hashimoto, K. Yoshida, S. NishiZaki, T. Fujita, J. G. Bednorz, and F. Lichtenberg, Nature (London) 372, 532 (1994).

${ }^{40}$ P. G. de Gennes, Superconductivity of Metals and Alloys (Benjamin, New York, 1966).

${ }^{41}$ C. Ishii, Prog. Theor. Phys. 44, 1525 (1970); C. Ishii, ibid. 47, 1646 (1972).

${ }^{42}$ J. Bardeen and J. L. Johnson, Phys. Rev. B 5, 72 (1972).

${ }^{43}$ A. F. Andreev, Zh. Eksp. Teor. Fiz. 46, 1823 (1964) [Sov. Phys. JETP 19, 1228 (1964)].

${ }^{44}$ We note that $T_{N}$ is the transmission probability at the Fermi energy in the superconducting state. In the $s$-wave junctions, $T_{N}$ is almost the same as the transmission probability in the normal 
state which is calculated from the normal conductance of junctions. In addition, $T_{N}$ is not sensitive to the energy of a quasiparticle around the Fermi level in the s-wave symmetry.

${ }^{45}$ I. O. Kulik and A. N. Omel'yanchuk, Fiz. Nizk. Temp. 3, 945 (1977) [Sov. J. Low Temp. Phys. 3, 459 (1977)].

${ }^{46}$ B. L. Al'tshuler and B. Z. Spivak, Pis'ma Zh. Eksp. Teor. Fiz. 65, 609 (1987) [JETP Lett. 65, 343 (1987)].

${ }^{47}$ Y. Koyama, Y. Takane, and H. Ebisawa, J. Phys. Soc. Jpn. 66, 430 (1997).

${ }^{48}$ In numerical simulations for the $d_{x y^{-}}$and $p_{y}$-wave symmetries, $\langle J\rangle$ does not become exactly zero. This is because higher harmonics [i.e., $J_{n} \sin (n \varphi)$ for $n \geq 2$ in Eq. (30)] contribute to $\langle J\rangle$. We con- firm that $|\langle J\rangle|$ is smaller than $\delta J$ by at least two orders of magnitude in the present simulation.

${ }^{49}$ I. O. Kulik and A. N. Omel'yanchuk, Pis'ma Zh. Eksp. Teor. Fiz. 21, 219 (1975) [JETP Lett. 21, 96 (1975)].

${ }^{50}$ The proximity effect is diagrammatically described by the socalled Cooperon propagator which has a singular behavior at $\omega_{n}=0$. The MARS is also characterized by the singular behavior of the Andreev reflection coefficients at $\omega_{n}=0$. The penetration of the MARS can be interpreted in terms of an interplay between the two singular behaviors in the Cooperon propagator and the Andreev reflection coefficients.

${ }^{51}$ Y. Tanaka and A. A. Golubov, cond-mat/0606231 (unpublished). 